\title{
Atrativo para abelhas em campos de produção de sementes de girassol colorido híbrido
}

\section{Honeybees attractant in hybrid colored sunflower seed production crop}

\author{
Emerson Augusto Castilho Martins ${ }^{1}$; Renato José Pires Machado²; José Lopes²*
}

\section{Resumo}

\begin{abstract}
A abelha Apis mellifera, em campos de produção de sementes híbridas de girassol (Helianthus annuus), apresenta preferência pelas plantas macho-estéreis, diminuindo a produção de sementes. Objetivando aumentar a visitação das abelhas nas plantas macho-estéreis, utilizou-se soluções de sacarose nas concentrações de $0,0 \%, 2,5 \%, 5,0 \%$ e 7,5\% (m/v), aplicadas em diferentes capítulos, numa área plantada com 1 ha de variedades ornamentais coloridas de girassol, em Londrina-PR. Foram utilizados 15 capítulos para cada tratamento, em 3 linhas internas à plantação. Após 2 horas da aplicação, realizou-se as contagens, caminhando-se nas ruas e anotando-se o número de abelhas nas flores marcadas. A maior média de visitação foi na concentração de $7,5 \%(x=0,43 \pm 0,11)$, não diferindo dos capítulos com solução de $5,0 \%$ $(x=0,42 \pm 0,08 ; p>0,05)$, mas com diferença significativa comparado estes dois tratamentos, controle $(x=0,12 \pm 0,04 ; p<0,05)$ e a $2,5 \%$ de concentração $(x=0,27 \pm 0,06 ; p>0,05)$. As abelhas africanizadas apresentaram uma resposta polinomial de segunda ordem para as concentrações testadas $(\hat{y}=0,11$ $\left.+8,40 \mathrm{x}-53,33 \mathrm{x}^{2} ; \mathrm{R}^{2}=0,9865\right)$. A aplicação de sacarose a 5,0\% foi capaz de aumentar em 4 vezes o número de visitação, abrindo perspectivas para novos estudos visando a adequada polinização de girassol $\operatorname{com} A$. mellifera como polinizador.
\end{abstract}

Palavras-chave: Apis mellifera, Helianthus annuus, sacarose, sementes, abelhas, girassol

\begin{abstract}
Africanized honeybee (Apis mellifera), in sunflower (Helianthus annuus) seed production crop, show male-fertile head preference, which decreases seed production. This research work aimed the honeybee visitation increase in male-sterile sunflower heads, with $0.0 \%, 2.5 \%, 5.0 \%$ and $7.5 \%(\mathrm{w} / \mathrm{v})$ sucrose solution, applied at sunflower heads in completely randomized design, on 1 ha seed crop production of colored ornamental sunflower strain, in Londrina County, Paraná State, Brazil. It was utilized 15 head for each treatment, on 3 rows internal to the crop. Two hours after the application, the number of honeybees was recorded walking through the rows and observing the number of bees on the marked flowers. The greatest visitation average was in $7.5 \%$ sugar dilution ( $x=0.43 \pm 0.11$, which was not different from $5.0 \%$ $(\mathrm{x}=0.42 \pm 0.08 ; \mathrm{p}>0.05)$ but significantly different from control heads $(\mathrm{x}=0.12 \pm 0.04 ; \mathrm{p}<0.05)$ and $2.5 \%$ sugar solution $(x=0.27 \pm 0.06 ; p>0.05)$. The number of africanized honeybees showed a $2^{\text {nd }}$ order polynomial response to the tested sugar solution $\left(\hat{y}=0,11+8,40 x-53,33 x^{2} ; R^{2}=0,9865\right)$. Sucrose $5.0 \%$ solution was capable to increase in four times the bee visitation, opening perspectives to new studies aiming adequate sunflower pollination with Africanized honeybees.
\end{abstract}

Key words: Apis mellifera, Helianthus annuus, sucrose, honeybee, sunflower

\footnotetext{
1 Programa de Pós-Graduação em Agronomia, Departamento de Agronomia, Universidade Estadual de Londrina; Departamento de Biologia,martinseac@yahoo.com.br

2 Departamento de Biologia Animal e Vegetal, Centro de Ciências Biológicas, Universidade Estadual de Londrina, Londrina, PR.

* Autor para correspondência.
} 


\section{Introdução}

A utilização da monocultura em larga escala, associado à aplicação de diferentes tipos de agroquímicos nas culturas atuais tem reduzido significativamente o número de agentes polinizadores nos agroecossistemas. Estas atividades, que visam garantir a produtividade, também são responsabilizadas por causar uma redução significativa na produção agrícola (DELAPLANE; MAYER, 2000). Perdas econômicas pela não introdução de polinizadores podem representar cerca de $600 \%$ sobre o custo da introdução (KEVAN; PHILLIPS, 2001).

No Brasil, a cultura de girassol (Helianthus annuus) para produção de sementes híbridas é realizada em plantações abertas, com polinização manual na maior parte das vezes. Este processo é demorado e dispendioso. Porém, no território brasileiro ocorre a abelha Apis mellifera (Linnaeus) africanizada, que é um híbrido das abelhas européias e africanas. Este híbrido é tido por Benedek e Maninguer (apud MORETI et al., 1996) como boa polinizadora de girassol, inclusive com maior fidelidade à visitação do que às abelhas européias (BASUALDO; BEDASCRRASBURE; DE JONG, 2000). Estes fatos levam muitos pesquisadores a estudarem a adição de colméias de abelhas africanizadas a plantações.

Porém, a real eficiência da A. mellifera é discutida por DeGrandi-Hoffman e Martin (1995) que realizaram a contagem de pólen no corpo das abelhas visitantes de linhas com capítulos macho-estéreis e concluíram que a abelha africanizada apresenta menos pólen em seu corpo que as demais espécies de abelhas coletadas. No Brasil, observações de campo corroboram com estes dados, já que é comum encontra-se abelhas visitando apenas capítulos macho-férteis.

Skinner (1987) ressalta que estudos devem ser realizados visando aumentar o movimento das abelhas entre as linhas macho-férteis e machos-estéreis na plantação, e Mahmood e Furgala (1983) observam que o retorno econômico da polinização entomófila é significante. Estudos foram realizados por Singh, Kumar e Chand (2000) para avaliar a atratividade de diferentes substâncias às abelhas nos capítulos de girassol. Deve-se considerar ainda a sugestão de Pham-Delegue et al. (1990) de que aparentemente a quantidade de abelhas está relacionado com a concentração de açúcar na planta. Realizou-se este trabalho com o objetivo de testar soluções de diferentes concentrações de sacarose como atrativo para abelhas africanizadas em produção de sementes híbridas.

\section{Material e Métodos}

O estudo foi realizado no mês de junho de 2002, na fazenda experimental da Embrapa (latitude 23 ${ }^{\circ} 11^{\prime}$ $\mathrm{S}$, longitude $51^{\circ} 11^{\prime} \mathrm{O}$ ), unidade CNPSo em Londrina, PR. A plantação ocupava uma área de 1 ha e destinava-se à produção de sementes híbridas de girassol ornamental colorido da cultivar BRS-OASIS.

No início do florescimento, no estádio $\mathrm{R}_{4}$ (floração inicial) (CASTIGLIONI et al., 1997), foram instaladas 3 colméias tipo Langstroth de abelhas africanizadas com população estabilizada e melgueira desprovida de mel.

Uma semana após a transferência das abelhas, quando os capítulos se encontravam na fase $R_{5.1}$, foram marcadas 15 plantas para cada um dos 3 tratamentos e mais 15 para o controle, totalizando 60 plantas. Na escolha das plantas, tomou-se o cuidado de escolher aquelas que apresentavam capítulos de tamanho homogêneo (aproximadamente $13 \mathrm{~cm}$ ) e voltados para as colméias, em 3 linhas, excluindo-se as 4 primeiras linhas para evitar efeito de borda.

Após três dias da introdução das colméias, quando os capítulos já se encontravam no estádio $\mathrm{R}_{5.1}$, com a abertura das primeiras flores no capítulo (CASTIGLIONI et al., 1997), iniciou-se o período de 4 dias seguidos de coleta de dados. Preparou-se, nestes quatro dias de observação, soluções de sacarose com $2,5 \%, 5,0 \%$ e $7,5 \%$ de concentração $(\mathrm{m} / \mathrm{v})$. Essas soluções foram aplicadas com 
pulverizador manual nas plantas marcadas às 13:30 horas. Contagens das abelhas visitantes nas flores durante os 4 dias foram realizadas após 2 horas da aplicação do atrativo (às 15:30 horas).

A contagem das abelhas era realizada visualmente, caminhando-se pelas ruas da plantação, sempre deixando duas linhas de distância entre o observador e as plantas observadas para não interferir no comportamento das abelhas. Registrou-se o número de abelhas pousadas sobre as flores no momento de visualização do capítulo pelo experimentador. Foram anotados somente dados de A. mellifera, desprezando-se outros visitantes florais.

Além do número de visitações de abelhas às flores, 50 dias após a fase de polinização, quando as sementes já se encontravam formadas e maduras, os capítulos marcados foram colhidos e levados ao laboratório para a contagem do número de sementes polinizadas.
Utilizou-se delineamento inteiramente casualizado. Os dados de contagem das abelhas foram transformados em $(x+0,5)^{1 / 2}$ devido ao alto número de capítulos sem nenhuma abelha, para as análises estatísticas. Realizou-se análise de regressão para verificação de possíveis padrões de atração das abelhas pelas flores pulverizadas. As médias foram comparadas pelo teste de Tukey (significância).

\section{Resultados}

A freqüência de abelhas visitando os capítulos onde haviam sido aplicados atrativos foi consideravelmente maior, e a correlação entre abelhas versus concentração da solução de sacarose apresentou-se como uma função quadrática, com a equação $\hat{y}=0,11+8,40 x-53,33 x^{2}$, obtendo-se um $R^{2}$ de $98,65 \%$ (Figura 1).

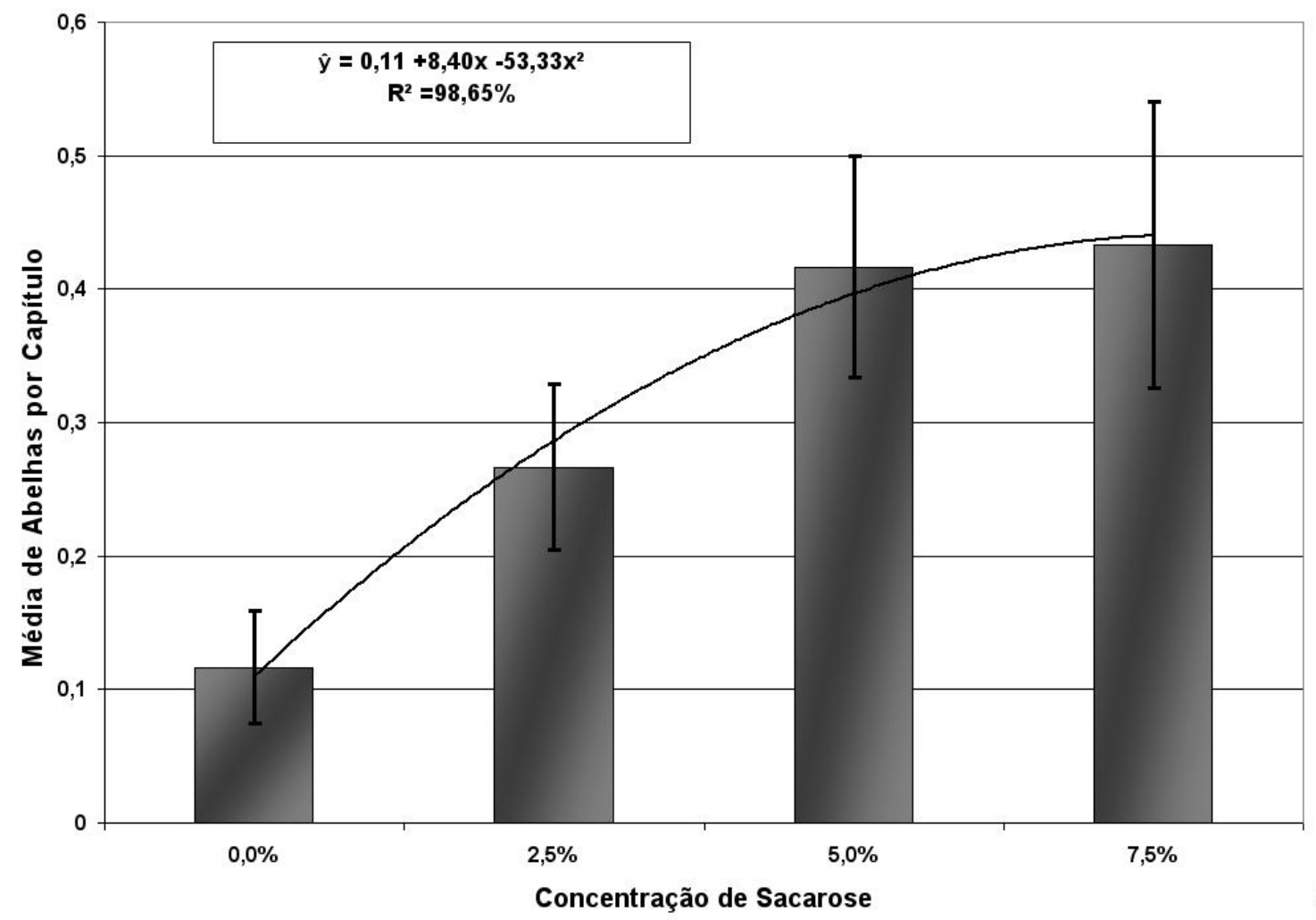

Figura 1. Correlação entre número de abelhas pousadas em capítulos macho-estéreis de girassol com aplicação de soluções de sacarose em diferentes concentrações, e respectivos erros-padrão, em junho de 2002, Londrina - PR. 
A diferença entre os tratamentos também foi observada através da análise de variância, seguida do teste de Tukey (Tabela 1). Observa-se diferenças significativas entre o controle, solução a $2,5 \%$ e $5,0 \%$. Os tratamentos de $5,0 \%$ e $7,5 \%$ não diferiram significativamente entre si (Tabela 2).
A média das sementes contadas nos capítulos coletados foi de apenas $6,95 \pm 1,19$ sementes. A análise de variância dos tratamentos é mostrada na Tabela 3.

Tabela 1. Análise de Variância do número de abelhas visitantes em capítulos de girassol macho-estéreis após a aplicação de soluções de sacarose como atrativos.

\begin{tabular}{lccc}
\hline Fonte da variação & gl & QM & F \\
\hline Tratamentos & 3,00 & 0,26 & $3,53^{*}$ \\
Resíduo & 236,00 & 0,07 & \\
Total & 239,00 & & \\
\hline Dados transformados $(\mathrm{X}+0,5)^{1 / 2}$ & $\mathrm{CV}=31,83 \%$ & $\mathrm{DMS}=0,0165$
\end{tabular}

Tabela 2. Média do número de abelhas visitantes em capítulos de girassol macho-estéreis após a aplicação de soluções de sacarose como atrativos. Letras iguais nas médias não diferem significativamente pelo teste de Tukey com um erro de $5,0 \%$.

\begin{tabular}{lcc}
\hline Tratamento & Soma & Média \\
\hline Controle & 46,05 & $0,77 \mathbf{a}$ \\
$2,5 \%$ & 50,55 & $0,84 \mathbf{b}$ \\
$5,0 \%$ & 54,56 & $0,91 \mathbf{c}$ \\
$7,5 \%$ & 54,19 & $0,90 \mathbf{c}$ \\
\hline
\end{tabular}

* Dados transformados em $(\mathrm{X}+0,5)^{1 / 2}$

Tabela 3. Análise de Variância do número de sementes em capítulos de girassol após a aplicação de soluções de sacarose como atrativos.

\begin{tabular}{lrcc}
\hline Fonte da variação & gl & QM & F \\
\hline Tratamentos & 3,00 & 1,28 & $0,82^{\text {NS }}$ \\
Resíduo & 36,00 & 1,57 & \\
Total & 39,00 & & \\
\hline
\end{tabular}




\section{Discussão}

Diferentes estudos confirmam a necessidade de introdução de polinizadores em agroecossistemas, já que o impacto da destruição de ecossistemas naturais e de uso de insumos tóxicos aos polinizadores é significativo (KEVAN; PHILLIPS, 2001). Porém, os dados disponíveis na literatura não permitem definir, com segurança, a quantidade adequada de abelhas necessárias para realizar uma eficiente polinização na produção de sementes de girassol. Embora existam trabalhos realizados em diversos locais do mundo com abelhas polinizando girassol, deve-se observar que estes dados nem sempre podem ser aplicados em território nacional: primeiro devido a presença em nosso território da abelha africanizada, um híbrido que apresenta diferenças significativas entre seleção de plantas quando comparada com a abelha européia utilizada na maioria dos estudos (BASUALDO; BEDASCRRASBURE; DE JONG, 2000); segundo porque o clima subtropical da região de Londrina apresenta diferenças muito grandes quando comparada com o clima temperado dos EUA e da Europa, locais onde a maior parte dos trabalhos encontrados são realizados.

Não houve correlação entre o número de visitas florais por abelhas e o número de sementes, o que contradiz os dados obtidos por Skinner (1987) e Rajagopal et al. (1999). Este resultado deve ter ocorrido devido ao número muito pequeno de $A$. mellifera encontradas no campo, associado ao fato de A. mellifera apresentar uma quantidade muito pequena de pólen em seu corpo se comparada com outros visitantes florais (De GRANDI-HOFFMAN; MARTIN, 1995). O fato do número de sementes granadas não ser grande pode ser observado quando se compara a média de sementes nos capítulos do experimento (6,95 sementes) pelo potencial total de produção de sementes, que pode chegar a 700 sementes. Devido a este baixo número, a polinização não foi adequada mesmo nos capítulos onde houve um maior número de visitas. Sugere-se que sejam realizados estudos com objetivo de avaliar a quantidade de colméias necessárias por ha na região, e que também se realizem trabalhos tentando associar A. mellifera com outras abelhas indígenas, que tendem a ser mais generalistas na coleta, discriminando menos entre capítulos macho-estéreis e macho-férteis. A quantidade de pólen no corpo de A. mellifera coletadas sobre capítulos machoestéreis está relacionada com o tamanho da população das abelhas não-Apis, mas a população de não-Apis é menor que de Apis, logo seria interessante a associação de ambas na plantação para uma possível obtenção de polinização adequada, que dispensasse a polinização manual (De GRANDIHOFFMAN; WATKINS, 2000).

Pequenas diferenças entre aromas de diferentes genótipos de plantas podem ser usadas pelas abelhas forrageiras como padrão discriminativo para a seleção da melhor fonte de alimento, o que deveria ser foco do interesse dos melhoristas na seleção de linhagens mais produtivas. Quando se leva em conta a capacidade de aprendizado de $A$. mellifera, pode-se esperar que elas respondam a uma gama de componentes menos específicos, como os compostos formados por moléculas grandes captados pelo tarso ou mesmo estímulos visuais, além dos voláteis da planta facilmente percebidos pelos insetos através das antenas. Logo, o estímulo olfativo tende então a ser mais importante no início da busca por alimento, pois parece que por um período mais prolongado, a recompensa alimentar poderá alterar o comportamento das abelhas (PHAM-DELEGUE et al., 1990). Talvez haja diferenças em aromas e compostos não-voláteis de capítulos macho-estéreis e macho-férteis, o que provocaria um comportamento que explique a tendência claramente observado no campo, tanto no Brasil quanto em outros países, das abelhas preferirem a visita aos capítulos férteis (SINGH; KUMAR; CHAND, 2000; SKINNER, 1987). Este problema agrava-se ainda com o fato de abelhas coletadas em capítulos macho-estéreis apresentarem uma menor quantidade de pólen em seu corpo do que outros visitantes florais (DE GRANDI-HOFFMAN; MARTIN, 1995). Um aumento do número de visitas poderia aumentar a 
produção (SKINNER, 1987; DE GRANDIHOFFMAN; WATKINS, 2000), e a discriminação entre macho-férteis e macho-estéreis poderia ser contornada através da aplicação de atrativos para abelhas, já que a discriminação entre macho-férteis e macho-estéreis pode estar relacionada com a concentração de açúcares no néctar das plantas (PHAM-DELEGUE et al., 1990). A aplicação de soluções de sacarose no presente experimento apresentou atratividade significativamente diferente, com um número de visitas quatro vezes maior que o controle quando se aplicou o atrativo. Quando comparamos resultados entre soluções a 5,0\% e a $7,5 \%$, não houve diferença significativa. $\mathrm{O}$ número de abelhas nos capítulos apresentou, na gama de concentrações testadas, um comportamento com tendência de função quadrática, com um $\mathrm{R}^{2}$ muito significativo (99\%) o que permite sugerir que aumentos na concentração do atrativo de 5,0\% a $7,5 \%$ não são vantajosos. Recomendam-se então estudos com a utilização de atrativos a base de solução de sacarose com concentração de 5,0\% em um campo saturado de abelhas, e também em campos onde haja interação de abelhas indígenas com as africanizadas.

\section{Referências}

BASUALDO, M.; BEDASCRRASBURE, E.; DE JONG, D. Africanized honey bees (Hymenoptera: Apidae) have a greater fidelity to sunflowers than European bees. Journal of Economic Entomology, Lanham, v.93, n.2, p.304-307. 2000.

CASTIGLIONI, V. B. R.; BALlA, A.; CASTRO, C.; SILVEIRA, J. M. Fases de desenvolvimento da planta de girassol. Londrina: EMBRAPA CNPSo, 1997. (Documentos, 58)

DE GRANDI-HOFFMAN, G.; MARTIN, J. H. Does a honey bee (Apis mellifera) colony's foraging population on malefertile sunflowers (Helianthus annuus) affect the amount of pollen on nestmates foraging on male-steriles? Journal of Apicultural Research, London, v.34, n.3, p.109-114, 1995.
DE GRANDI-HOFFMAN, G.; WATKINS, J. C. The foraging activity of honey bees Apis mellifera and nonApis bees on hybrid sunflower (Helianthus annuus) and its influence on cross-pollination and seed set. Journal of Apicultural Research, London, v.39, n.1, p.37-45, 2000.

DELAPLANE, K. S.; MAYER, E. D. F. Crop pollination by bees. New York: CABI, 2000.

KEVAN, P. G.; PHILLIPS, T. P. The economic impacts of pollinator declines: an approach to assessing the consequences. Conservation Ecology, Ontario, v.5, n.1, art.8, 2001. Disponível em: <http://www.consecol.org/vol5/ iss $1 /$ art8>. Acesso em: 5 jun. 2001.

MAHMOOD, A. N.; FURGALA, B. Effect of pollination by insects on seed oil percentage of oilseed sunflower. American Bee Journal, Hamilton, v.123, n.9, p.663-667, 1983.

MORETI, A. C. C. C.; SILVA, R. M. B.; SILVA, E. C. A.; ALVES, M. L. T. M. F.; OTSUK, I. P. Aumento na produção de sementes de girassol (Helianthus annuus) pela ação de insetos polinizadores. Scientia Agrícola, Piracicaba, v.53, n.2-3, p.280-284, 1996.

PHAM-DELEGUE, M. H.; ETIEVANT, P.; GHUICHARD, E.; MARILLEAU, R.; DOUALT, P. H.; CHAUFFAILLE, J.; MASSON, C. Chemicals involved in honeybee-sunflower relationship. Journal of Chemical Ecology, New York, v.16, n.11, p.3053-3065, 1990.

RAJAGOPAL, D.; VEERESH, G. K.; CHIKKADEVAIAH, NAGARAJA, N; KENCHARADDI, R.N. Potentiality of honeybees in hybrid seed production of sunflower (Helianthus annuus). Indian Journal of Agricultural Sciences, New Delhi, v.69, n.1, p.40-43, 1999.

SINGH, R.; KUMAR, S.; CHAND, H. Increasing the attractiveness of sunflower to honeybees for pollination. Shashpa: Journal of Entomology, Oman, v.7, n.2, p.151$154,2000$.

SKINNER, J. A. Abundance and spatial distribution of bees visiting male-sterile and male-fertile sunflower cultivars in Califórnia. Environmental Entomology, Maryland (College Park), v.16, n.4, p.922-927, 1987. 